Predictive Science Academic Alliances Program (PSAAP) Technical White Paper Turbulent Mixing and Hydrodynamics

O. Schilling, M. Steinkamp, M. Baer

February 24, 2006 
This document was prepared as an account of work sponsored by an agency of the United States Government. Neither the United States Government nor the University of California nor any of their employees, makes any warranty, express or implied, or assumes any legal liability or responsibility for the accuracy, completeness, or usefulness of any information, apparatus, product, or process disclosed, or represents that its use would not infringe privately owned rights. Reference herein to any specific commercial product, process, or service by trade name, trademark, manufacturer, or otherwise, does not necessarily constitute or imply its endorsement, recommendation, or favoring by the United States Government or the University of California. The views and opinions of authors expressed herein do not necessarily state or reflect those of the United States Government or the University of California, and shall not be used for advertising or product endorsement purposes.

This work was performed under the auspices of the U.S. Department of Energy by University of California, Lawrence Livermore National Laboratory under Contract W-7405-Eng-48. 


\title{
Predictive Science Academic Alliances Program (PSAAP) Technical White Paper \\ Turbulent Mixing and Hydrodynamics
}

\author{
Oleg Schilling, Lawrence Livermore National Laboratory \\ Michael J. Steinkamp, Los Alamos National Laboratory \\ Melvin R. Baer, Sandia National Laboratory
}

February 2006

\begin{abstract}
The design of efficient, high-gain capsules for inertial confinement fusion (ICF) ${ }^{1}$ and the modeling of supernova implosions and explosions ${ }^{2}$ requires a detailed understanding of the consequences of material interpenetration, hydrodynamic instabilities and mixing at molecular (or atomic) scales arising from perturbations at material interfaces, i.e., the Rayleigh-Taylor, Richtmyer-Meshkov and Kelvin-Helmholtz instabilities (buoyancy-, shock- and shear-induced instabilities, respectively). From a computational point of view, this requires the development of models for hydrodynamic instability growth from initial perturbations through the weakly- and strongly-nonlinear phases, and finally, to the latetime turbulent regime. In particular, modeling these processes completely and accurately is critical for demonstrating the feasibility and potential success of contemporary ICF capsule designs. A predictive computational capability for the effects of turbulent mass, momentum, energy and species transport, as well as material mixing, on the thermonuclear fusion process in ICF entails the development of turbulent transport and mixing or subgrid-scale models based on statistically-averaged or filtered evolution equations, respectively. The former models are typically referred to as Reynoldsaveraged Navier-Stokes (RANS) (and related) models ${ }^{3}$ and the latter are referred to as large-eddy simulation (LES) models. ${ }^{4}$ The strong nonlinearity of the equations describing the hydrodynamics, thermodynamics, material properties and other multi-scale phenomena, together with the formal ensemble averaging or filtering procedure, introduce correlations of strongly-fluctuating fields and other a priori unclosed quantities that must be explicitly modeled to close the set of equations describing the implosion dynamics and burning of an ICF capsule.
\end{abstract}

\section{Overview}

The Advanced Simulation and Computing (ASC) Program is pursuing a multidisciplinary research effort to develop and quantitatively validate turbulent transport and mixing models using numerical simulations, theoretical modeling and experimental data. Specifically, it is of interest to develop state-of-the-art theoretical and numerical approaches for modeling the hydrodynamics and turbulent mixing generated by hydrodynamic instabilities induced by interfacial perturbations. A related objective is to establish the infrastructure needed to extract quantities of interest (e.g., model quantities and flow visualizations) from large-scale direct numerical simulations (DNS) ${ }^{5}$ and from 
large-eddy or monotone-integrated (or implicit) large-eddy simulations (MILES or ILES). ${ }^{6}$ Numerical simulations in support of model development and a priori or $a$ posteriori validation consider the spatio-temporal evolution of turbulent mixing ${ }^{7}$ induced by hydrodynamic interfacial instabilities (and possibly other types of compressible single- or multi-fluid flows) on parallel computing platforms. Such data can be used to address the proper modeling of initial conditions in averaged descriptions of turbulent mixing, and to examine in detail the transition from the linear to the nonlinear and to the turbulent growth regimes. It is highly desirable to relate this work to experimental data, when available. Hence, a goal is to address the physical and mathematical formulation of turbulence models, with an emphasis on the principal unclosed quantities that must be modeled using gradient diffusion and other potentially more sophisticated and physicallycomplete closures (e.g., second-moment closures ${ }^{8}$ ). Higher order closure models provide variances that are directly related to mixing properties, and which are not available from first-order closure models. Another goal is to analyze the intrinsic properties of numerical algorithms used to solve systems of equations containing either embedded turbulent transport and mixing models or explicit/implicit subgrid-scale models.

Presently, eddy viscosity modeling methods, LES/MILES/ILES methods, and kinetic (particle) models are the methods of choice to model turbulent flows. However, the complex nonlinear physics of turbulence, as well as the proper mathematical and numerical (discrete) representation of that physics, continue to be open research areas. The ASC program is interested in research in these areas that can be applied to the computational modeling of turbulence.

\section{Turbulent Mixing}

The ASC Program requires models describing hydrodynamics and turbulence over an extensive range of spatio-temporal scales, including the development of hydrodynamic interfacial instabilities (including the Rayleigh-Taylor, Richtmyer-Meshkov and KelvinHelmholtz instabilities) from small-amplitude linear growth to weakly- and stronglynonlinear growth, and finally to the late-time fully-developed turbulent regime. This includes the development of DNS capability and of subgrid-scale models for LES of turbulent mixing. ${ }^{7}$ The models should provide detailed information regarding concentration profiles, and the distribution of, and correlations among, the sizes and shapes of regions of unmixed or poorly mixed fluid. It is also necessary to develop improved constitutive closure models for the Reynolds stresses and other unclosed quantities for use in RANS models, possibly using data from DNS, LES and MILES/ILES. Research into alternatives to conventional subgrid-scale models, such as models based on filters and sub-filters, is also beneficial. Conceptual issues such as explicit filtering in complex geometries and on unstructured grids for inhomogeneous turbulence should be addressed.

The simulations should attain very large Reynolds numbers characteristic of fullydeveloped turbulence and mixing. DNS resolves all of the spatial and temporal scales in the flow, whereas LES resolves only the largest scales while modeling the averaged effects of the unresolved (smaller) scales on the resolved scales. DNS is challenging, as turbulent mixing layers generated by interfacial instabilities can grow from very smallscale perturbations and a quiescent state, implying a very wide range of spatial scales. 
Note that conventional DNS of shock-driven flows is not possible, due to the inability to fully resolve shocks. LES is also challenging, as there is a need to model both the subgrid-scale terms in the large-eddy equations (the explicitly or implicitly filtered conservation equations) and the unresolved initial conditions. MILES and ILES rely instead on implicit subgrid-scale models inherent in the numerical discretization of the non-dissipative fluid dynamics equations, and do not require explicit subgrid-scale models. However, such simulations do not exhibit pointwise convergence and may be illposed in multiple dimensions; the small scales are determined by the regularizing numerical dissipation, so that the mixing properties depend on the spatial resolution and on other properties of the algorithm. Additional research is needed in assessing the applicability of different MILES and ILES methods for simulating turbulent mixing.

Phenomenological or rigorous mathematical models are needed for the dependence of turbulent mixing properties on such conditions as the initial density and velocity, the material density ratio at a sharp interface, the direction and history of the acceleration, the compressibility of the fluid, and the initial values of symmetry-breaking perturbations (such as density and velocity fluctuations, and surface roughness). These models are needed in forms suitable for numerical discretization, and it is particularly desirable that they be implemented and evaluated as RANS or subgrid-scale models within much more coarsely-zoned simulations than required to perform DNS.

High-fidelity turbulence modeling is of interest to predictively model unsteady, highlynonequilibrium, inhomogeneous, anisotropic, variable-density and fully-compressible multi-fluid turbulence. The transition of unstable flows to turbulence and the dependence of the mixing layer properties on the initial conditions are of interest. The development of models and codes that can incorporate the closed equations governing the Reynolds stresses and other second-order quantities, and which predict the dynamics and statistical properties of evolving hydrodynamic instabilities are sought.

Variable-density and compressibility effects ${ }^{9}$ are important in all of the applications mentioned above. In particular, it is important to understand the effects of shocks of various strengths on turbulence and on turbulent mixing layers, including the effects on coherent structures and on the structures resulting from the evolution of the instabilities through baroclinic effects within the mixing layer. Models must incorporate buoyancy, shock interactions and backscatter effects (energy transfer from small to larger scales due to nonlinear interactions).

Turbulent combustion, ${ }^{10}$ other exothermic chemical reactions and thermonuclear reactions must be accounted for and understood in the presence of all of the aforementioned phenomena. Combustion and related processes are particularly challenging, as they occur at molecular and atomic scales, which are unresolved on any possible spatio-temporal computational grid. Consequently, probabilistic descriptions ${ }^{11}$ have traditionally been used to model the average effects in reacting flows. The understanding of combustion and related phenomena is most useful if it spans a large range of ratios of chemical energy release to the rates of other energetic processes, such as the conversion of instability energy into turbulence and the conversion of turbulent kinetic energy into internal energy. Note that MILES and ILES methods are problematic 
for simulating combustion, as their inherent dissipation is likely to be insufficient to adequately model the subgrid-scale dynamics. ${ }^{12}$

Whenever possible, numerical models of all of these processes should be tested and validated against high-resolution DNS or experimental data.

\section{Hydrodynamics}

The ASC Program requires the solution of a broad range of hydrodynamics problems, ranging from low-speed subsonic flows to shocked supersonic flows. The physical and mechanical phenomena of interest include turbulence and turbulent mixing, material strength, high-displacement and high-strain rate flows, and highly distorted hydrodynamic flows with shocks. Three-dimensional multi-scale methods capable of modeling such phenomena over a broad range of length- and timescales and relevant dimensionless parameters are needed. Some problems of interest require the ability to track shock fronts. As the flow domains of interest can be geometrically complex, unstructured grid algorithms compatible with high-aspect ratio zoning are highly desirable.

A variety of methods have previously been used to model flows of interest, including finite-difference, finite-volume, finite-element and particle techniques. These methods can be purely Eulerian, purely Lagrangian or hybrid. Examples of hybrid methods are adaptive mesh refinement (AMR), which can model shock propagation on an Eulerian grid, and arbitrary Lagrangian-Eulerian (ALE) methods. While single-fluid flows are of interest, multiple-fluid/multi-phase flows are particularly relevant.

Needed algorithm research includes: (1) determining the relative strengths, weaknesses, and domains of applicability of a wide variety of hydrodynamics algorithms (such an effort might develop test problems, some with analytic or semi-analytic solutions that can be used to evaluate algorithms); (2) evaluation of the behavior of these algorithms in physics codes which model hydrodynamic flows and turbulence, transport, material strength and other effects, and; (3) development of new computational fluid dynamics algorithms to improve upon deficient algorithms. For example, numerical analysis in support of turbulence and subgrid-scale model development and assessment could quantitatively analyze simulation data and apply analytical techniques (e.g., modified equation or truncation error analysis) to estimate the effects of the numerical errors and other components of an algorithm on the properties of the solutions, i.e., intrinsic numerical dissipation. Such research is essential, as the numerical algorithms used in either RANS or LES models cannot be developed independently of the models, e.g., it is important to understand the competition between the inherent numerical dissipation in the algorithm and the dissipation modeled in the turbulence model. Issues such as estimating the errors associated with a subgrid-scale model near the Nyquist scale imposed by the grid resolution and the relationship between the grid and filter scales should be addressed for the flows of interest.

It is essential to compare simulation data with a comprehensive set of hydrodynamic experiments which complement the algorithm development and evaluation. 


\section{References}

${ }^{1}$ Nakai, S. \& Takabe, H. 1996 Principles of inertial confinement fusion-physics of implosion and the concept of inertial fusion energy. Reports on Progress in Physics 59 1071-1131; Lindl, J. D. 1999 Inertial Confinement Fusion: The Quest for Ignition and Energy Gain Using Indirect Drive, Springer-Verlag; Atzeni, S. \& Meyer-ter-Vehn, J. 2004 The Physics of Inertial Fusion: Beam Plasma Interaction, Hydrodynamics, Hot Dense Matter, Oxford University Press.

${ }^{2}$ Arnett, W. D. 1987 Supernova Theory and Supernova 1987A. Astrophysical Journal 319, 136-142; Arnett, W. D., Fryxell, B. A. \& Müller, E. 1989 Instabilities and Nonradial Motion in SN-1987A. Astrophysical Journal 341, L63-L66; Chevalier, R. A., Blondin, J. M. \& Emmering, R. T. 1992 Hydrodynamic Instabilities in Supernova Remnants: Self-Similar Driven Waves. Astrophysical Journal 392, 118-130; Kimoto, P. A. \& Chernoff, D. F. 1997 Radiative Instabilities in Simulations of Spherically Symmetric Supernova Blast Waves. Astrophysical Journal 485, 274-284; Kane, J., Drake, R. P. \& Remington, B. A. 1999 An Evaluation of the Richtmyer-Meshkov Instability in Supernova Remnant Formation. Astrophysical Journal 511, 335-340.

${ }^{3}$ Speziale, C. G. 1998 Turbulence modeling for time-dependent RANS and VLES: A review. AIAA Journal 36, 173-184; Gatski, T. B. 2004 Constitutive equations for turbulent flows. Theoretical and Computational Fluid Dynamics 18, 345-369.

${ }^{4}$ Lesieur, M. \& Métais, O. 1996 New Trends in Large-Eddy Simulations of Turbulence. Annual Review of Fluid Mechanics 28, 45-82; Spalart, P. R. 2000 Strategies for turbulence modelling and simulations. International Journal of Heat and Fluid Flow 21, 252-263; Moin P. 2002 Advances in large eddy simulation methodology for complex flows. International Journal of Heat and Fluid Flow 23, 710-720; Sagaut, P. 2005 Large Eddy Simulation for Incompressible Flows: An Introduction, second edition, Springer-Verlag.

${ }^{5}$ Moin, P. \& Mahesh, K. 1998 Direct Numerical Simulation: A Tool in Turbulence Research. Annual Review of Fluid Mechanics 30, 539-578.

${ }^{6}$ Boris, J. P., Grinstein, F. F., Oran, E. S. \& Kolbe, R. L. 1992 New Insights into Large Eddy Simulation. Fluid Dynamics Research 10, 199-228; Hahn M. \& Drikakis, D. 2005 Large eddy simulation of compressible turbulence using high-resolution methods. International Journal for Numerical Methods in Fluids 47, 971-977; Margolin, L. G. \& Rider, W. J. 2005 The design and construction of implicit LES models. International Journal for Numerical Methods in Fluids 47, 1173-1179.

${ }^{7}$ Dimotakis, P. E. 2005 Turbulent Mixing. Annual Review of Fluid Mechanics 37, 329-356.

${ }^{8}$ Speziale, C. G. 1991 Analytical Methods for the Development of Reynolds-Stress Closures in Turbulence. Annual Review of Fluid Mechanics 23, 107-157; Jaw, S.-Y. \& Chen, C.-J. 1998 Present Status of Second-Order Closure Turbulence Models. I: Overview. Journal of Engineering Mechanics 124, 485-501; Chen, C.-J. \& Jaw, S.-Y. 1998 Fundamentals of Turbulence Modeling, Taylor and Francis; Leschziner, M. A. 2001 Statistical Turbulence Modelling for the Computation of Physically Complex Flows. New Trends in Turbulence, Proceedings of the Les Houches Summer Session Vol. 74, edited by M. Lesieur, A. Yaglom, F. David, 187, SpringerVerlag.

${ }^{9}$ Chassaing, P., Antonia, R. A. \& Anselmet, F., Joly, L. \& Sarkar, S., 2002 Variable Density Fluid Turbulence, Fluid Mechanics and its Applications Vol. 69, Kluwer Academic.

${ }^{10}$ Peters, N. Turbulent Combustion, Cambridge University Press.

${ }^{11}$ Fox, R. O. 2003 Computational Models for Turbulent Reacting Flows, Cambridge University Press.

${ }^{12}$ Pitsch, H. 2006 Large-Eddy Simulation of Turbulent Combustion. Annual Review of Fluid Mechanics 38,453-482. 